\title{
Ensemble modeling of stochastic unsteady open-channel flow in terms of its time-space evolutionary probability distribution - Part 1: theoretical development
}

\author{
Alain Dib and M. Levent Kavvas \\ Department of Civil and Environmental Engineering, University of California, Davis, 95616, USA
}

Correspondence: Alain Dib (aedib@ucdavis.edu)

Received: 4 July 2017 - Discussion started: 9 August 2017

Accepted: 5 March 2018 - Published: 28 March 2018

\begin{abstract}
The Saint-Venant equations are commonly used as the governing equations to solve for modeling the spatially varied unsteady flow in open channels. The presence of uncertainties in the channel or flow parameters renders these equations stochastic, thus requiring their solution in a stochastic framework in order to quantify the ensemble behavior and the variability of the process. While the Monte Carlo approach can be used for such a solution, its computational expense and its large number of simulations act to its disadvantage. This study proposes, explains, and derives a new methodology for solving the stochastic Saint-Venant equations in only one shot, without the need for a large number of simulations. The proposed methodology is derived by developing the nonlocal Lagrangian-Eulerian FokkerPlanck equation of the characteristic form of the stochastic Saint-Venant equations for an open-channel flow process, with an uncertain roughness coefficient. A numerical method for its solution is subsequently devised. The application and validation of this methodology are provided in a companion paper, in which the statistical results computed by the proposed methodology are compared against the results obtained by the Monte Carlo approach.
\end{abstract}

\section{Introduction}

Unsteady open-channel flows are a common occurrence in hydrology and hydraulics problems. They arise as a result of the movement of water waves in natural or artificial channels (Sturm, 2001). Understanding and tracing the movement of such water waves along the channels is of great importance in addressing engineering flow problems, including flood forecasting, flood control, hydrograph generation, and several others (Chow, 1959). The technique used to approximate and trace such water waves is known as flood routing, and the governing equations that are commonly used to solve for the unsteady flows in flood routing problems are known as the Saint-Venant equations (Chanson, 2004).

Various uncertainties may add to the complexity of solving the Saint-Venant equations (Gates and AlZahrani, 1996a; Ercan and Kavvas, 2012a) and these may correspond to several factors. Physical conditions of open channels may be uncertain due to their high degree of variability (Sturm, 2001). One example is Manning's roughness coefficient, which greatly depends on the channel vegetation, bed material, bedforms, and even on the position of the free water surface (Chow, 1959; Sturm, 2001; Ercan and Kavvas, 2012a). With the uncertainties in quantifying or characterizing these factors, the roughness coefficient becomes extremely difficult to estimate (Sturm, 2001), rendering it uncertain. Channel geometric parameters may also be uncertain. This includes the channel bed slope (Ercan and Kavvas, 2012a) and the channel cross section geometry, the latter of which may exhibit significant spatial variability across a river due to its irregular form and due to the changes it may undergo along the direction of flow (Chow, 1959). Other uncertainties may also arise from lateral inflows and initial conditions due to their spatial and/or temporal variability (Liang and Kavvas, 2008), as well as from the upstream boundary conditions due to the temporal variability of the inflows into the channel.

As a result of such uncertainties, the channel and flow parameters may be considered spatially and/or temporally random at the local scale of a river cross section, rendering 
the system behavior uncertain (Gates and AlZahrani, 1996a). Therefore, deterministically solving the Saint-Venant equations in this case would no longer be providing a representative solution to the flood routing problem being considered. In fact, in this study the governing partial differential equations (PDEs), i.e., the Saint-Venant equations, will be transformed into stochastic PDEs because the channel and flow parameters are now stochastic and can be described as random functions (Liang and Kavvas, 2008). This means that the dependent variables that will be solved for by these equations (e.g., flow velocity and depth) will also be spatiotemporal random functions. Hence, instead of solving for the deterministic values of the dependent variables, the goal will be to solve for their statistical properties (Van Kampen, 1976), which can be obtained at designated discrete time-space positions.

Two popular methods can be used for such a stochastic solution to nonlinear problems: the finite-order analysis and the Monte Carlo (MC) approach. Applying the expectation operator, or any other statistical moment operator, to nonlinear difference equations with stochastic parameters may result in nonlinear expressions that are difficult, or even impossible, to simplify into terms involving the known moments of the model parameters and the unknown moments of the dependent variables (Gates and AlZahrani, 1996a). Finite-order analysis overcomes this by performing a Taylor series expansion of the difference equations about the expected values of the parameters, from which higher-order terms are truncated. For example, truncating the Taylor series after the firstorder term is known as the first-order approximation, which is a good approximation when the system nonlinearity is not too high, and when the stochastic parameters have relatively small coefficients of variation (Dettinger and Wilson, 1981). However, with highly nonlinear problems, instead of using higher-order approximations of the finite-order method, it may be required and more efficient to use full-distribution methods such as the MC approach (Dettinger and Wilson, 1981).

The MC approach is well-known for simulating differential equations with stochastic parameters, and is used to determine the distributions of the unknown stochastic dependent variables (Freeze, 1975; Smith and Freeze, 1979; Bellin et al., 1992). This method involves repeatedly solving the governing equations in a deterministic fashion, varying the stochastic parameters for each run, in order to obtain a set of several realizations for each of the dependent variables. When a sufficient number of realizations is obtained, they can be used to determine the required statistical properties, including the mean system behavior and the standard deviation (Gates and AlZahrani, 1996b). Therefore, the MC simulations require two models: one which generates realizations for the stochastic parameters, and another (finite-difference model) which deterministically solves the governing flow equations for each realization (Gates and AlZahrani, 1996b). The MC approach is generally accepted as the most robust approach for uncertainty evaluation, as well as the benchmark for comparing other new methods (Scharffenberg and Kavvas, 2011). The full distribution characteristics may be estimated using the MC approach, which is more intuitive than the finite-order methods (Gates and AlZahrani, 1996a). However, the main drawback of the MC approach is its computational expense due to the usual running of a large number of simulations of the process under study in order to obtain accurate results (Dettinger and Wilson, 1981).

To bypass the need for solving the unsteady open-channel flow governing equations several times, a new methodology is proposed in this study in order to solve for the expected system behavior and variability in only one simulation. This methodology involves upscaling the governing stochastic differential equations from the point scale (at which they are originally valid) to the field scale. Ensemble averaging has been a common approach to upscale hydrologic equations that are linear (Gelhar and Axness, 1983; Kitanidis, 1988; Rubin and Dagan, 1989; Kapoor and Gelhar, 1994; Kavvas and Karakas, 1996; Wood and Kavvas, 1999a, b) or nonlinear (Mantoglou and Gelhar, 1987; Tayfur and Kavvas, 1994; Horne and Kavvas, 1997; Dogrul et al., 1998), in which case these equations are averaged to become deterministic differential equations. These developed deterministic differential equations use statistical descriptions, such as the mean and variance, to represent the values of the stochastic parameters (Liang and Kavvas, 2008). However, most of the studies performing the ensemble averaging technique on nonlinear conservation equations used the regular perturbation method, which includes linearization assumptions and which only works for small fluctuations in the dependent variables (Kavvas, 2003). Other techniques that have been applied, which are not limited by small fluctuations, include the decomposition method (Serrano, 1995), a combination of volume averaging with nonlinear dynamics (Duffy, 1996; Duffy and Cusumano, 1998), as well as the theory of fractals and multifractals (Puente, 1996). Nonetheless, due to some limitations of such methods when used for stochastic nonlinear hydrologic processes, the upscaling method used in this study is chosen to be that of Kavvas (2003).

Kavvas (2003) developed general ensemble average conservation equations (to second order) for nonlinear and linear hydrologic processes in order to determine their probabilistic and mean behavior. The "master key" equations developed may be used on any stochastic hydrologic process after being rewritten as one or more linear/nonlinear stochastic ordinary differential equations (ODEs). This utilization leads to a special Lagrangian-Eulerian form of the Fokker-Planck equation (LEFPE) that models the time-space evolution of the probability density of the dependent variables of any nonlinear/linear stochastic dynamic process (Kavvas, 2003). Such a methodology has been successfully applied to many hydrologic processes, including unsaturated water flow (Kim et al., 2005b), root-water uptake (Kim et al., 2005a), solute transport (Liang and Kavvas, 2008), snow accumulation and 
melt (Ohara et al., 2008), unconfined groundwater flow (Cayar and Kavvas, 2009a, b), and kinematic open-channel flow (Ercan and Kavvas, 2012a, b).

Noting that the characteristic forms of the Saint-Venant equations are nonlinear ODEs, it is proposed in this study to apply to them their corresponding master key equation from Kavvas (2003). From this operation the corresponding LEFPE of the Saint-Venant open-channel flow equations is obtained, thus providing the ability to model the uncertainties of the channel and flow parameters and to compute their effect on the behavior of the system. Therefore, under the appropriate initial and boundary conditions, the probability density functions (PDFs) of the dependent variables can be computed (to exact second order) through the LEFPE, and the ensemble behavior of the system can be described.

The advantages of using the LEFPE in tackling the flood routing problem greatly echo those of the classical FokkerPlanck equation (FPE). In fact, the LEFPE directly solves for the PDFs of the dependent variables of the system in both time and space, it is linear in the variable being solved for (i.e., in the PDF), and unlike the many simulations usually performed for the MC approach, the LEFPE produces the complete ensemble model results with only one single simulation. As such, the LEFPE provides not only the mean and variance of the process but also a complete description of the evolution of the dependent variables' PDFs in a computationally efficient manner. Note that the LEFPE does not make any linearization assumptions, it works with a wide-ranging parameter space, and the only assumption about the physical process it makes is the finite correlation time for the process (Ercan and Kavvas, 2012a).

Therefore, following from the above discussion, the main objective of this study is to apply the upscaling method based on the LEFPE approach in Kavvas (2003) to the characteristic form of the stochastic Saint-Venant equations in order to derive a new methodology that solves for the probability density of the dependent flow variables, and that quantifies the expected behavior and variability of the system in one shot, instead of running a large number of simulations.

\section{Saint-Venant equations for unsteady open-channel flow}

The Saint-Venant equations, also known as the spatially varied unsteady flow equations (Sturm, 2001), are the two governing equations used to describe an unsteady open-channel flow problem that will be solved using the hydraulic routing technique (Chow, 1959; Viessman et al., 1977; Sturm, 2001). They consist of the continuity equation and the momentum equation which are used simultaneously in order to solve for the two unknowns (velocity and depth, or discharge and depth). The naming of these equations comes from the French mathematician Adhémar-Jean-Claude Barré de Saint-Venant who published the equations describing one- dimensional unsteady open-channel flow in 1871 (Barré de Saint-Venant, 1871).

Several assumptions are made when deriving these equations (Viessman et al., 1977; Sturm, 2001), including unidirectional flow and uniform cross-sectional velocity, hydrostatic pressure, small channel bed slope, steady state estimation of friction loss, and incompressible flow. Following these assumptions, the Saint-Venant equations for unsteady open-channel flow of an incompressible fluid in a rectangular, prismatic channel (with no lateral inflow/outflow) may be written as follows (Viessman et al., 1977):

$$
\begin{aligned}
& \text { Continuity } y \frac{\partial V}{\partial x}+V \frac{\partial y}{\partial x}+\frac{\partial y}{\partial t}=0, \\
& \text { Momentum } \frac{\partial V}{\partial t}+V \frac{\partial V}{\partial x}+g \frac{\partial y}{\partial x}=g\left(S_{0}-S_{\mathrm{f}}\right),
\end{aligned}
$$

where $V$ is the average flow velocity, $y$ is the flow depth, $x$ is the position, $t$ is the time, $S_{0}$ is the slope of the channel bottom, $S_{\mathrm{f}}$ is the friction slope, and $g$ is the acceleration of gravity. When these assumptions are no longer valid, the derived Saint-Venant equations will show some limitations. Such limitations occur, for example, if the flow is not one-dimensional (e.g., in flood plains or large rivers), if the pressure is non-hydrostatic (e.g., presence of sharp geometric variations/bends or hydraulic jumps), if there are sharp discontinuities (e.g., those caused by weirs or gates), or if there are channel irregularities (Litrico and Fromion, 2009). Therefore, keeping those limitations in mind is crucial for the appropriate implementation of the derived Saint-Venant equations.

\subsection{Solution methods for the Saint-Venant equations}

Since closed-form solutions to the Saint-Venant equations have not been obtained due to the presence of nonlinear terms, it has not been possible to solve these equations analytically except when extreme simplifications are applied (Sturm, 2001; Chaudhry, 2008). As a result, several numerical techniques have been developed in order to solve the Saint-Venant equations deterministically in their full form, without major simplifications. The most frequently used of these techniques are finite-difference methods (Abbott and Ionescu, 1967; Fread, 1973; Beam and Warming, 1976; Fennema and Chaudhry, 1986; Garcia and Kahawita, 1986; Venutelli, 2002), which solve the governing equations explicitly or implicitly along a fixed or adaptive $x-t$ grid (Szymkiewicz, 2010). Finite-element methods are also available for solving such unsteady flow equations (Cooley and Moin, 1976; Szymkiewicz, 1991, 1995; Hicks and Steffler, 1995), though they are usually considered to be more effective for two- and three-dimensional flow problems (Szymkiewicz, 1991). While the standard finite-element method may not be the most suitable or satisfactory method for solving unsteady flow problems (Szymkiewicz, 2010), the modified finite-element method (Szymkiewicz, 1995) 
seems to be as effective and robust in solving such problems as the high-order finite-difference methods.

Furthermore, another approach to solve the Saint-Venant equations may be utilized after realizing that these equations are hyperbolic PDEs (Chaudhry, 2008). This approach is known as the method of characteristics (MOC; Abbott, 1966), which is one of the earliest and most exact methods for solving hyperbolic PDEs (Tannehill et al., 1997), and which was used early on for solving the Saint-Venant equations (Amein, 1966; Woolhiser and Liggett, 1967; Lai, 1988). The MOC may be used to transform a hyperbolic PDE into a system of ODEs, which may be simpler to solve (Sturm, 2001). These ODEs are usually divided into two equations: the characteristic equation (i.e., the ODE describing the characteristic path), and the compatibility equation (i.e., the ODE that describes the process behavior along that characteristic path) (Hoffman, 2001). After this transformation by the MOC, the finite-difference approximations of the derivatives can then be applied to the characteristic form of the hyperbolic PDE, instead of applying them to its original form. Note that in time and one-space dimensions, the characteristic equations represent curves in the $x-t$ plane along which information propagates through the solution domain (Hoffman, 2001), and along which discontinuities in the derivatives of the dependent variables propagate (Sturm, 2001).

From the several techniques available to solve for the Saint-Venant equations, the MOC is chosen for this study. This is because, as was mentioned in Sect. 1, the upscaling technique based on the LEFPE approach in Kavvas (2003) can be applied to hydrologic processes that are written as one or more ODEs. As such, it is imperative for the progression of this study to transform the Saint-Venant equations into their characteristic form in order to write them as a system of ODEs. With two characteristic directions, the SaintVenant equations are transformed by the MOC into a system of four ODEs: two characteristic equations and two corresponding compatibility equations. When finite-difference approximations are applied to the characteristic form of the Saint-Venant equations, the results can be numerically computed along an irregular $x-t$ grid formed by the intersection points of the characteristic curves (Gates and AlZahrani, 1996a).

\subsection{Characteristic form of the Saint-Venant equations}

Through a linear combination of the continuity and momentum equations (Eqs. 1 and 2), the characteristic equations for unsteady open-channel flow of an incompressible fluid in a rectangular, prismatic channel with no lateral inflow can be written as follows (Sturm, 2001):

Positive characteristic curve $\left(C_{1}\right)$

$\frac{\mathrm{d} x_{1}}{\mathrm{~d} t}=V+c$.
Flow process condition to be satisfied along $C_{1}$

$\left(\frac{\mathrm{d}(V+2 c)}{\mathrm{d} t}\right)_{1}=g\left(S_{0,1}-S_{\mathrm{f}, 1}\right)$.

Negative characteristic curve $\left(C_{2}\right)$

$\frac{\mathrm{d} x_{2}}{\mathrm{~d} t}=V-c$.

Flow process condition to be satisfied along $C_{2}$

$\left(\frac{\mathrm{d}(V-2 c)}{\mathrm{d} t}\right)_{2}=g\left(S_{0,2}-S_{\mathrm{f}, 2}\right)$,

where $c$ is the wave celerity which is equal to $\sqrt{g y}$ for a rectangular channel, and $S_{0,2}$ is equal to $S_{0}\left(x_{2}, t\right)$ (similarly for the other $S$ variables). The remaining variables are defined as in Eqs. (1) and (2).

Equations (3) and (5) represent two different velocity expressions defining the two characteristic directions of the Saint-Venant equations: the former defining the positive characteristic curve $\left(C_{1}\right)$ with speed $V+c$, and the latter defining the negative characteristic curve $\left(C_{2}\right)$ with speed $V-c$. Equations (4) and (6) represent the compatibility equations for Eqs. (3) and (5), respectively. Each compatibility equation for the flow process behavior should be satisfied along its corresponding characteristic curve. The subscripts in Eqs. (3) to (6) are used to differentiate between the two total derivative operators which correspond to the two different speeds along $C_{1}$ and $C_{2}$. As such, Eqs. (3) to (6) are seen to describe the change of two functions along two different paths: a function $V+2 c$ that varies along $C_{1}$, and another function $V-2 c$ that varies along $C_{2}$. These functions are known as the Riemann invariants (Chaudhry, 2008).

Therefore, the MOC transforms the two governing PDEs into a system of four ODEs that are differentiated with respect to time only, and no longer with respect to space. This transformation provides the ability to use the upscaling technique based on the LEFPE approach in Kavvas (2003) on the Saint-Venant equations in order to derive this study's proposed methodology involving the ensemble-averaged equations of stochastic unsteady open-channel flow.

\section{Ensemble-averaged equations for the stochastic unsteady open-channel flow}

In this section, a new methodology for solving the stochastic Saint-Venant equations will be introduced and derived, and a numerical discretization scheme will be devised for it as well. The proposed methodology aims at obtaining the statistical properties of the dependent variables of the unsteady open-channel flow system in only one simulation, as opposed to the large number of simulations usually involved in the MC approach. The following derivation involves assuming the Manning's roughness coefficient as an uncertain parameter, but similar steps can be followed even when the uncertainty is assumed to arise from other parameters. 


\subsection{Development of the Fokker-Planck solution methodology for the Saint-Venant equations}

Following Kavvas (2003), a system of point-scale conservation equations can be written for a dynamical system as follows:

$$
\frac{\partial \boldsymbol{H}(\boldsymbol{x}, t)}{\partial t}=\eta(\boldsymbol{H}, \mathbf{A}, \boldsymbol{f} ; \boldsymbol{x}, t),
$$

where $\boldsymbol{H}(\boldsymbol{x}, t)$ is the vector of all state variables of the hydrologic system of equations; $\mathbf{A}(\boldsymbol{x}, t)$ is the tensor of parameters; $\boldsymbol{f}(\boldsymbol{x}, t)$ is the vector of forcing functions; $\boldsymbol{\eta}$ is a function of $\boldsymbol{H}, \mathbf{A}$, and $\boldsymbol{f} ; \boldsymbol{x}$ is the vector of spatial locations, and $t$ is the time. The initial condition for the above system is given as

$\boldsymbol{H}(\boldsymbol{x}, 0)=\boldsymbol{H}_{0}$.

Under a second-order cumulant expansion, the general Lagrangian-Eulerian form of the Fokker-Planck equation (LEFPE) for the previously defined dynamical system was developed in Kavvas (2003, Eq. 25) to exact second order (i.e., to the order of the covariance time of $\eta$ ) as shown below:

$$
\begin{aligned}
& \frac{\partial P\left(\boldsymbol{H}\left(\boldsymbol{x}_{t}, t\right) ; t\right)}{\partial t}=-\frac{\partial}{\partial H_{j}}\left\{P\left(\boldsymbol{H}\left(\boldsymbol{x}_{t}, t\right) ; t\right)\right. \\
& \left.\left[\begin{array}{c}
\left\langle\eta_{j}\left(\boldsymbol{H}\left(\boldsymbol{x}_{t}, t\right), \mathbf{A}\left(\boldsymbol{x}_{t}, t\right), \boldsymbol{f}\left(\boldsymbol{x}_{t}, t\right)\right)\right\rangle \\
+\int_{0}^{t} \mathrm{~d} s \operatorname{Cov}_{\mathrm{O}}\left[\frac{\partial \eta_{j}\left(\boldsymbol{H}\left(\boldsymbol{x}_{t}, t\right), \mathbf{A}\left(\boldsymbol{x}_{t}, t\right), \boldsymbol{f}\left(\boldsymbol{x}_{t}, t\right)\right)}{\partial H_{i}} ;\right. \\
\left.\eta_{i}\left(\boldsymbol{H}\left(\boldsymbol{x}_{t-s}, t-s\right), \mathbf{A}\left(\boldsymbol{x}_{t-s}, t-s\right), \boldsymbol{f}\left(\boldsymbol{x}_{t-s}, t-s\right)\right)\right]
\end{array}\right]\right\} \\
& +\frac{1}{2} \frac{\partial^{2}}{\partial H_{j} \partial H_{i}}\left\{\begin{array}{c}
t \\
2 P\left(\boldsymbol{H}\left(\boldsymbol{x}_{t}, t\right) ; t\right) \int_{0}^{t} \mathrm{~d} s \operatorname{Cov}_{\mathrm{o}}\left[\eta _ { j } \left(\boldsymbol{H}\left(\boldsymbol{x}_{t}, t\right),\right.\right. \\
\left.\mathbf{A}\left(\boldsymbol{x}_{t}, t\right), \boldsymbol{f}\left(\boldsymbol{x}_{t}, t\right)\right) ; \eta_{i}\left(\boldsymbol{H}\left(\boldsymbol{x}_{t-s}, t-s\right), \mathbf{A}\left(\boldsymbol{x}_{t-s}, t-s\right),\right. \\
\left.\left.\left.\boldsymbol{f}\left(\boldsymbol{x}_{t-s}, t-s\right)\right)\right]\right\}
\end{array}\right.
\end{aligned}
$$

where $P\left(\boldsymbol{H}\left(\boldsymbol{x}_{t}, t\right) ; t\right)$ is the probability density function of the vector of state variables $(\boldsymbol{H})$ at location $\boldsymbol{x}_{t}$ and at time $t$, the operator $\langle\bullet\rangle$ is the ensemble average operator, $s$ is a time displacement, and $\operatorname{Cov}_{\mathrm{o}}[\bullet]$ is the time-ordered covariance function as shown in the below equation (Van Kampen, 1974):

$$
\begin{aligned}
\operatorname{Cov}_{\mathrm{o}}\left[\eta_{j}\left(\boldsymbol{x}, t_{1}\right) ; \eta_{i}\left(\boldsymbol{x}, t_{2}\right)\right] & =\left\langle\eta_{j}\left(\boldsymbol{x}, t_{1}\right) \eta_{i}\left(\boldsymbol{x}, t_{2}\right)\right\rangle \\
& -\left\langle\eta_{j}\left(\boldsymbol{x}, t_{1}\right)\right\rangle\left\langle\eta_{i}\left(\boldsymbol{x}, t_{2}\right)\right\rangle .
\end{aligned}
$$

Note that in Eq. (9), the real space location $\boldsymbol{x}_{t}$ is known, whereas the Lagrangian location $\boldsymbol{x}_{t-s}$ is unknown. This Lagrangian location can be determined from the known location $\boldsymbol{x}_{t}$ by using a Lie operator as defined in Kavvas and Karakas (1996):

$\boldsymbol{x}_{t-s}=\overleftarrow{\exp }\left[-\int_{t-s}^{t} \mathrm{~d} \tau\left\langle v_{l}\left(\boldsymbol{x}_{\tau}, \tau\right)\right\rangle \frac{\partial}{\partial x_{l}}\right] \boldsymbol{x}_{t}$, where $\overleftarrow{\exp }$ is the time-ordered exponential, and $v_{l}$ is determined from the characteristic curve equation corresponding to a particular hydrologic/hydraulic conservation equation. In the three-dimensional flow case, $l$ takes on the values $1-$ 3. A first-order approximation of Eq. (11) can be written as follows (Kavvas and Karakas, 1996):

$\boldsymbol{x}_{t-s}=\boldsymbol{x}_{t}-\int_{t-s}^{t} \mathrm{~d} \tau\left\langle\boldsymbol{v}\left(\boldsymbol{x}_{\tau}, \tau\right)\right\rangle$,

where $\boldsymbol{v}=\left[v_{1}, v_{2}, v_{3}\right]$ in the general three-dimensional flow case.

Solving the LEFPE, Eq. (9), under the appropriate initial and boundary conditions provides the spatiotemporal evolution of the PDF of the vector of state variables $(\boldsymbol{H})$ for any hydrologic system expressed in terms of Eqs. (7) and (8), thus providing the ensemble behavior and variability of the process with only one simulation. In addition, it is important to note that the LEFPE, a parabolic PDE, is a deterministic equation that is linear in its unknown variable $P\left(\boldsymbol{H}\left(\boldsymbol{x}_{t}, t\right)\right.$, $t$ ), unlike the original hydrologic system which would usually be stochastic and nonlinear. As such, the LEFPE provides great advantages in simplifying the stochastic solution of the hydrologic system being considered.

Since the LEFPE was developed for a system of ODEs (Eq. 7), and since the characteristic form of the Saint-Venant equations is a system of four nonlinear ODEs (Eqs. 3 to 6), it is proposed to apply to these equations the corresponding LEFPE after making some substitutions and adjustments. First, the friction slope $\left(S_{\mathrm{f}}\right)$ is computed using Manning's formula (Sturm, 2001). Then, the Riemann invariants are defined as follows:

$V+2 c=\alpha$,

$V-2 c=\beta$.

As such, Eqs. (3) to (6) can be written as a system of four ODEs in terms of four state variables $\left(x_{1}, x_{2}, \alpha, \beta\right)$ :

$$
\begin{aligned}
& \frac{\mathrm{d} x_{1}}{\mathrm{~d} t}=\frac{3}{4} \alpha\left(x_{1}, t\right)+\frac{1}{4} \beta\left(x_{1}, t\right) \equiv \eta_{1, t}\left(x_{1}, t\right)=\eta_{1, t} \\
& \begin{aligned}
\left(\frac{\mathrm{d} \alpha}{\mathrm{d} t}\right)_{1} & =g S_{0}\left(x_{1}, t\right)-\frac{g n^{2}\left(x_{1}, t\right)}{k^{2}} \cdot \frac{\frac{1}{4}\left[\alpha\left(x_{1}, t\right)+\beta\left(x_{1}, t\right)\right]^{2}}{R^{4 / 3}\left(\alpha\left(x_{1}, t\right), \beta\left(x_{1}, t\right), b ; x_{1}, t\right)} \\
& \equiv \eta_{\alpha, t}\left(x_{1}, t\right)=\eta_{\alpha, t}
\end{aligned} \\
& \begin{aligned}
\frac{\mathrm{d} x_{2}}{\mathrm{~d} t}= & \frac{1}{4} \alpha\left(x_{2}, t\right)+\frac{3}{4} \beta\left(x_{2}, t\right) \equiv \eta_{2, t}\left(x_{2}, t\right)=\eta_{2, t} \\
\left.\frac{\mathrm{d} \beta}{\mathrm{d} t}\right)_{2} & =g S_{0}\left(x_{2}, t\right)-\frac{g n^{2}\left(x_{2}, t\right)}{k^{2}} \cdot \frac{\frac{1}{4}\left[\alpha\left(x_{2}, t\right)+\beta\left(x_{2}, t\right)\right]^{2}}{R^{4 / 3}\left(\alpha\left(x_{2}, t\right), \beta\left(x_{2}, t\right), b ; x_{2}, t\right)} \\
& \equiv \eta_{\beta, t}\left(x_{2}, t\right)=\eta_{\beta, t}
\end{aligned}
\end{aligned}
$$

where $R$ denotes the hydraulic radius, $n$ denotes Manning's roughness coefficient, and $k$ denotes the conversion factor between SI and US units for Manning's formula. Note that 
the width of the channel $(b)$ is not a function of $x$ or $t$ since the equations are derived for a prismatic channel. Moreover, $g$ and $k$ are also not functions of $x$ or $t$ since they are constants. Equations (15) to (18) are now in the form of Eq. (7), with an $\eta$ function defined for each of the four ODEs, as shown on their right-hand side. As such, it is clear that for a vector of state variables $\boldsymbol{H}=\left[x_{1}, x_{2}, \alpha, \beta\right]$, there is a vector of functions $\boldsymbol{\eta}=\left[\eta_{1, t}, \eta_{2, t}, \eta_{\alpha, t}, \eta_{\beta, t}\right]$. The subscripts for the $\eta$ functions shown in Eqs. (15) to (18) represent the following: subscript 1 represents the positive characteristic direction $\left(C_{1}\right)$, subscript 2 represents the negative characteristic direction $\left(C_{2}\right)$, subscript $\alpha$ represents the compatibility equation along $C_{1}$, and subscript $\beta$ represents the compatibility equation along $C_{2}$.

Note that in equations to follow, the $\eta$ functions will be interchangeably represented by one of their two forms shown on the right-hand side of Eqs. (15) to (18), whereas $P\left(x_{1}\right.$, $\left.x_{2}, \alpha, \beta, t\right)$ may be substituted by $P$ for simplicity. Such typographical simplifications will be used in order to reduce the used space and to increase the readability and simplicity of large equations. Therefore, considering some of the above typographical simplifications, and applying the general LEFPE in Eq. (9) to Eqs. (15) to (18), the LEFPE for the Saint-Venant equations that would solve for the multivariate PDF of the hydrologic state vector can be written to exact second order as shown in Eq. (19) below.

$$
\begin{aligned}
& \frac{\partial P\left(x_{1}, x_{2}, \alpha, \beta ; t\right)}{\partial t}= \\
& -\frac{\partial}{\partial x_{1}}\left\{P\left(x_{1}, x_{2}, \alpha, \beta ; t\right)\left[\begin{array}{c}
\left\langle\eta_{1, t}\left(x_{1}, t\right)\right\rangle \\
+\int_{0}^{t} \mathrm{~d} s \operatorname{Cov}_{\mathrm{o}}\left[\frac{\partial \eta_{1, t}\left(x_{1}, t\right)}{\partial x_{1}} ; \eta_{1, t-s}\left(x_{1}, t-s\right)\right] \\
+\int_{0}^{t} \mathrm{~d} s \operatorname{Cov}_{\mathrm{o}}\left[\frac{\partial \eta_{1, t}\left(x_{1}, t\right)}{\partial x_{2}} ; \eta_{2, t-s}\left(x_{2}, t-s\right)\right] \\
+\int_{0}^{t} \mathrm{~d} s \operatorname{Cov}_{\mathrm{o}}\left[\frac{\partial \eta_{1, t}\left(x_{1}, t\right)}{\partial \alpha} ; \eta_{\alpha, t-s}\left(x_{1}, t-s\right)\right] \\
+\int_{0}^{t} \mathrm{~d} s \operatorname{Cov}_{\mathrm{o}}\left[\frac{\partial \eta_{1, t}\left(x_{1}, t\right)}{\partial \beta} ; \eta_{\beta, t-s}\left(x_{2}, t-s\right)\right]
\end{array}\right]\right\} \\
& -\frac{\partial}{\partial x_{2}}\left\{P\left(x_{1}, x_{2}, \alpha, \beta ; t\right)\left[\begin{array}{c}
\left\langle\eta_{2, t}\left(x_{2}, t\right)\right\rangle \\
+\int_{0}^{t} \mathrm{~d} s \operatorname{Cov}_{\mathrm{o}}\left[\frac{\partial \eta_{2, t}\left(x_{2}, t\right)}{\partial x_{2}} ; \eta_{2, t-s}\left(x_{2}, t-s\right)\right] \\
+\int_{0}^{t} \mathrm{~d} s \operatorname{Cov}_{\mathrm{o}}\left[\frac{\partial \eta_{2, t}\left(x_{2}, t\right)}{\partial x_{1}} ; \eta_{1, t-s}\left(x_{1}, t-s\right)\right] \\
+\int_{0}^{t} \mathrm{~d} s \operatorname{Cov}_{\mathrm{o}}\left[\frac{\partial \eta_{2, t}\left(x_{2}, t\right)}{\partial \alpha} ; \eta_{\alpha, t-s}\left(x_{1}, t-s\right)\right] \\
+\int_{0}^{t} \mathrm{~d} s \operatorname{Cov}_{\mathrm{o}}\left[\frac{\partial \eta_{2, t}\left(x_{2}, t\right)}{\partial \beta} ; \eta_{\beta, t-s}\left(x_{2}, t-s\right)\right]
\end{array}\right]\right\} \\
& -\frac{\partial}{\partial \alpha}\left\{P\left(x_{1}, x_{2}, \alpha, \beta ; t\right)\left[\begin{array}{c}
\left\langle\eta_{\alpha, t}\left(x_{1}, t\right)\right\rangle \\
+\int_{0}^{t} \mathrm{~d} s \operatorname{Cov}_{\mathrm{o}}\left[\frac{\partial \eta_{\alpha, t}\left(x_{1}, t\right)}{\partial \alpha} ; \eta_{\alpha, t-s}\left(x_{1}, t-s\right)\right] \\
+\int_{0}^{t} \mathrm{~d} s \operatorname{Cov}_{\mathrm{o}}\left[\frac{\partial \eta_{\alpha, t}\left(x_{1}, t\right)}{\partial x_{1}} ; \eta_{1, t-s}\left(x_{1}, t-s\right)\right] \\
+\int_{0}^{t} \mathrm{~d} s \operatorname{Cov}_{0}\left[\frac{\partial \eta_{\alpha, t}\left(x_{1}, t\right)}{\partial x_{2}} ; \eta_{2, t-s}\left(x_{2}, t-s\right)\right] \\
+\int_{0}^{t} \mathrm{~d} s \operatorname{Cov}_{0}\left[\frac{\partial \eta_{\alpha, t}\left(x_{1}, t\right)}{\partial \beta} ; \eta_{\beta, t-s}\left(x_{2}, t-s\right)\right]
\end{array}\right]\right\}
\end{aligned}
$$

$$
\begin{aligned}
& -\frac{\partial}{\partial \beta}\left\{P\left(x_{1}, x_{2}, \alpha, \beta ; t\right)\left[\begin{array}{c}
\left\langle\eta_{\beta, t}\left(x_{2}, t\right)\right\rangle \\
+\int_{0}^{t} \mathrm{~d} s \operatorname{Cov}_{\mathrm{o}}\left[\frac{\partial \eta_{\beta, t}\left(x_{2}, t\right)}{\partial \beta} ; \eta_{\beta, t-s}\left(x_{2}, t-s\right)\right] \\
+\int_{0}^{t} \mathrm{~d} s \operatorname{Cov}_{\mathrm{o}}\left[\frac{\partial \eta_{\beta, t}\left(x_{2}, t\right)}{\partial x_{1}} ; \eta_{1, t-s}\left(x_{1, t-s}, t-s\right)\right] \\
+\int_{0}^{t} \mathrm{~d} s \operatorname{Cov}_{\mathrm{o}}\left[\frac{\partial \eta_{\beta, t}\left(x_{2}, t\right)}{\partial x_{2}} ; \eta_{2, t-s}\left(x_{2}, t-s\right)\right] \\
+\int_{0}^{t} \mathrm{~d} s \operatorname{Cov}_{\mathrm{o}}\left[\frac{\partial \eta_{\beta, t}\left(x_{2}, t\right)}{\partial \alpha} ; \eta_{\alpha, t-s}\left(x_{1, t-s}, t-s\right)\right]
\end{array}\right]\right\} \\
& +\frac{1}{2} \frac{\partial^{2}}{\partial x_{1}^{2}}\left\{2 P\left(x_{1}, x_{2}, \alpha, \beta ; t\right) \int_{0}^{t} \mathrm{~d} s \operatorname{Cov}_{\mathrm{o}}\left[\eta_{1, t}\left(x_{1}, t\right) ; \eta_{1, t-s}\left(x_{1}, t-s\right)\right]\right\} \\
& +\frac{1}{2} \frac{\partial^{2}}{\partial x_{2}^{2}}\left\{2 P\left(x_{1}, x_{2}, \alpha, \beta ; t\right) \int_{0}^{t} \mathrm{~d} s \operatorname{Cov}_{\mathrm{o}}\left[\eta_{2, t}\left(x_{2}, t\right) ; \eta_{2, t-s}\left(x_{2}, t-s\right)\right]\right\} \\
& +\frac{1}{2} \frac{\partial^{2}}{\partial \alpha^{2}}\left\{2 P\left(x_{1}, x_{2}, \alpha, \beta ; t\right) \int_{0}^{t} \mathrm{~d} s \operatorname{Cov}_{\mathrm{o}}\left[\eta_{\alpha, t}\left(x_{1}, t\right) ; \eta_{\alpha, t-s}\left(x_{1}, t-s\right)\right]\right\} \\
& +\frac{1}{2} \frac{\partial^{2}}{\partial \beta^{2}}\left\{2 P\left(x_{1}, x_{2}, \alpha, \beta ; t\right) \int_{0}^{t} \mathrm{~d} s \operatorname{Cov}_{\mathrm{o}}\left[\eta_{\beta, t}\left(x_{2}, t\right) ; \eta_{\beta, t-s}\left(x_{2}, t-s\right)\right]\right\} \\
& + \text { other cross-covariance dispersion terms. }
\end{aligned}
$$

Note that the LEFPE has the form of an advectiondiffusion equation. In Eq. (19), the first four terms on the right-hand side represent the advection terms, while the remaining terms represent the diffusion terms. Within the advection terms, the expected values of the $\eta$ functions are the mean advection coefficients, while the integrals of the ordered covariance functions added to them are the advection correction terms. However, it was shown in a study by Kavvas and $\mathrm{Wu}$ (2002), which used a similar approach but was applied to solute transport, that the advection correction terms are negligible when compared to the mean advection term. As a result, the magnitudes of the expectations of the $\eta$ functions are much larger than those of the integral terms in the advection portion of the LEFPE, thus allowing the removal of these integral terms. As an example, this simplification can be mathematically represented for the first advection term as shown in Eq. (20) below, and is similarly applied to the other advection terms.

$$
\begin{aligned}
\left\langle\eta_{1, t}\right\rangle & \gg \operatorname{Cov}_{\mathrm{O}}\left[\frac{\partial \eta_{1, t}}{\partial x_{1}} ; \eta_{1, t-s}\right], \\
\left\langle\eta_{1, t}\right\rangle & \gg \operatorname{Cov}_{\mathrm{O}}\left[\frac{\partial \eta_{1, t}}{\partial \alpha} ; \eta_{\alpha, t-s}\right], \\
\left\langle\eta_{1, t}\right\rangle & \gg \operatorname{Cov}_{\mathrm{O}}\left[\frac{\partial \eta_{1, t}}{\partial x_{2}} ; \eta_{2, t-s}\right], \\
\left\langle\eta_{1, t}\right\rangle & \gg \operatorname{Cov}_{\mathrm{O}}\left[\frac{\partial \eta_{1, t}}{\partial \beta} ; \eta_{\beta, t-s}\right] .
\end{aligned}
$$

Moreover, note that the cross-covariance dispersion terms have not been explicitly written in Eq. (19). These terms involve the ordered covariance between two different $\eta$ functions, $\eta_{i}$ and $\eta_{j}$, where $i \neq j$. Examples of those include:

$\operatorname{Cov}_{\mathrm{o}}\left[\eta_{1, t}\left(x_{1}, t\right) ; \eta_{2, t-s}\left(x_{2}, t-s\right)\right]$, 
$\operatorname{Cov}_{\mathrm{o}}\left[\eta_{1, t}\left(x_{1}, t\right) ; \eta_{\beta, t-s}\left(x_{2}, t-s\right)\right]$.

However, all of the four $\eta$ functions $\left(\eta_{1, t} ; \eta_{2, t} ; \eta_{\alpha, t} ; \eta_{\beta, t}\right)$ are functions of the state stochastic variables $\left(x_{1} ; x_{2} ; \alpha ; \beta\right)$. It has been shown in Liang and Kavvas (2008) that the covariance between any two of the different $\eta$ functions is substantially smaller in magnitude when compared to the autocovariance of the $\eta$ function of any one state variable. This leads to another simplification in which all cross-covariance terms, similar to those in Eq. (21), are neglected and removed from the main equation. Such an approximation may break down when the functions are similar, in the sense of their behavior or even the existence of periodicity with close frequencies. In such cases, the cross-covariance terms of the two $\eta$ functions may become closer in magnitude to the autocovariance values, possibly invalidating their neglection. As a result of these simplifications, Eq. (19) can be written as shown in Eq. (22).

$$
\begin{aligned}
& \frac{\partial P\left(x_{1}, x_{2}, \alpha, \beta ; t\right)}{\partial t}=-\frac{\partial}{\partial x_{1}}\left\{P\left\langle\eta_{1, t}\left(x_{1}, t\right)\right\rangle\right\} \\
& +\frac{1}{2} \frac{\partial^{2}}{\partial x_{1}^{2}}\left\{2 P \int_{0}^{t} \mathrm{~d} s \operatorname{Cov}_{\mathrm{o}}\left[\eta_{1, t}\left(x_{1}, t\right) ; \eta_{1, t-s}\left(x_{1}, t-s\right)\right]\right\} \\
& -\frac{\partial}{\partial x_{2}}\left\{P\left\langle\eta_{2, t}\left(x_{2}, t\right)\right\rangle\right\} \\
& +\frac{1}{2} \frac{\partial^{2}}{\partial x_{2}^{2}}\left\{2 P \int_{0}^{t} \mathrm{~d} s \operatorname{Cov}_{\mathrm{o}}\left[\eta_{2, t}\left(x_{2}, t\right) ; \eta_{2, t-s}\left(x_{2}, t-s\right)\right]\right\} \\
& -\frac{\partial}{\partial \alpha}\left\{P\left\langle\eta_{\alpha, t}\left(x_{1}, t\right)\right|\right\} \\
& +\frac{1}{2} \frac{\partial^{2}}{\partial \alpha^{2}}\left\{2 P \int_{0}^{t} \mathrm{~d} s \operatorname{Cov}_{\mathrm{o}}\left[\eta_{\alpha, t}\left(x_{1}, t\right) ; \eta_{\alpha, t-s}\left(x_{1}, t-s\right)\right]\right\} \\
& -\frac{\partial}{\partial \beta}\left\{P\left\langle\eta_{\beta, t}\left(x_{2}, t\right)\right|\right\} \\
& +\frac{1}{2} \frac{\partial^{2}}{\partial \beta^{2}}\left\{2 P \int_{0}^{t} \mathrm{~d} s \operatorname{Cov}_{\mathrm{o}}\left[\eta_{\beta, t}\left(x_{2}, t\right) ; \eta_{\beta, t-s}\left(x_{2}, t-s\right)\right]\right\}
\end{aligned}
$$

Until this point, there has been no approximation regarding the covariance expressions in the diffusion coefficients of Eq. (22). However, note that the $\eta$ functions describe motions occurring in opposite directions, in a similar manner to how $\alpha$ may describe a forward propagation motion and $\beta$ may describe a backward propagation motion, based on their equations. Such backward and forward propagation directions would be expected to have a relatively weak correlation. As a result of this, and as a result of the order of magnitude analysis performed in Liang and Kavvas (2008), one final simplification can be applied to the LEFPE. This simplification involves the approximation that the $\eta_{i}$ random functions have short memory with respect to $t$, and thus may be approximated as delta-correlated. As a result, the covariance integral term for any of the four functions can be written as follows (where $\delta(s)$ is the Dirac delta function):

$$
\begin{aligned}
\int_{0}^{t} \mathrm{~d} s \operatorname{Cov}_{\mathrm{o}}\left[\eta_{i, t} ; \eta_{i, t-s}\right] & =\int_{0}^{t} \operatorname{Cov}_{\mathrm{o}}\left[\eta_{i, t} ; \eta_{i, t-s}\right] \delta(s) \mathrm{d} s \\
& =\operatorname{Var}\left[\eta_{i, t}\right] .
\end{aligned}
$$

Note that under the approximation of a delta-correlated covariance, the nonlocal LEFPE reduces to the classical FPE (as it will be called henceforth), which is simpler to apply. Including all of the simplifications discussed above, Eq. (19) can be written as shown in Eq. (24).

$$
\begin{aligned}
& \frac{\partial P\left(x_{1}, x_{2}, \alpha, \beta ; t\right)}{\partial t}= \\
& -\frac{\partial}{\partial x_{1}}\left\{P\left\langle\eta_{1, t}\left(x_{1}, t\right)\right\rangle\right\}+\frac{1}{2} \frac{\partial^{2}}{\partial x_{1}^{2}}\left\{2 P \operatorname{Var}\left[\eta_{1, t}\left(x_{1}, t\right)\right]\right\} \\
& -\frac{\partial}{\partial x_{2}}\left\{P\left\langle\eta_{2, t}\left(x_{2}, t\right)\right\rangle\right\}+\frac{1}{2} \frac{\partial^{2}}{\partial x_{2}^{2}}\left\{2 P \operatorname{Var}\left[\eta_{2, t}\left(x_{2}, t\right)\right]\right\} \\
& -\frac{\partial}{\partial \alpha}\left\{P\left\langle\eta_{\alpha, t}\left(x_{1}, t\right)\right\rangle\right\}+\frac{1}{2} \frac{\partial^{2}}{\partial \alpha^{2}}\left\{2 P \operatorname{Var}\left[\eta_{\alpha, t}\left(x_{1}, t\right)\right]\right\} \\
& -\frac{\partial}{\partial \beta}\left\{P\left\langle\eta_{\beta, t}\left(x_{2}, t\right)\right\rangle\right\}+\frac{1}{2} \frac{\partial^{2}}{\partial \beta^{2}}\left\{2 P \operatorname{Var}\left[\eta_{\beta, t}\left(x_{2}, t\right)\right]\right\}
\end{aligned}
$$

The validity of the preceding approximations will be checked when the results of the proposed FPE methodology are compared against the corresponding results obtained from the MC approach. With the final version of the FPE being ready, the last step is to determine the detailed expressions of the expectations and variances of the $\eta$ functions in Eq. (24). After expanding these expressions based on their equivalence as denoted in Eqs. (15) to (18), and after some manipulation, Eq. (24) may be written as follows:

$$
\begin{aligned}
& \frac{\partial P\left(x_{1}, x_{2}, \alpha, \beta ; t\right)}{\partial t}=-\frac{\partial}{\partial x_{1}}\left\{P\left[\frac{3}{4}\left\langle\alpha\left(x_{1}, t\right)\right\rangle+\frac{1}{4}\left\langle\beta\left(x_{1}, t\right)\right\rangle\right]\right\} \\
& -\frac{\partial}{\partial x_{2}}\left\{P\left[\frac{1}{4}\left\langle\alpha\left(x_{2}, t\right)\right\rangle+\frac{3}{4}\left\langle\beta\left(x_{2}, t\right)\right\rangle\right]\right\} \\
& -\frac{\partial}{\partial \alpha}\left\{P \left[g S_{0}-\frac{g}{4 k^{2}}\left(\frac{2}{b}\right)^{4 / 3}\left\langle n^{2}\left(x_{1}, t\right) \cdot\left[\alpha\left(x_{1}, t\right)+\beta\left(x_{1}, t\right)\right]^{2}\right.\right.\right. \\
& \left.\left.\left.\cdot\left\{\frac{8 g b}{\left[\alpha\left(x_{1}, t\right)-\beta\left(x_{1}, t\right)\right]^{2}}+1\right\}^{4 / 3}\right\rangle\right]\right\} \\
& -\frac{\partial}{\partial \beta}\left\{P \left[g S_{0}-\frac{g}{4 k^{2}}\left(\frac{2}{b}\right)^{4 / 3}\left\langle n^{2}\left(x_{2}, t\right) \cdot\left[\alpha\left(x_{2}, t\right)+\beta\left(x_{2}, t\right)\right]^{2}\right.\right.\right. \\
& \left.\left.\left.\cdot\left\{\frac{8 g b}{\left[\alpha\left(x_{2}, t\right)-\beta\left(x_{2}, t\right)\right]^{2}}+1\right\}^{4 / 3}\right\rangle\right]\right\} \\
& +\frac{\partial^{2}}{\partial x_{1}^{2}}\left\{P \left[\left(\frac{9}{16}\right) \operatorname{Var}\left[\alpha\left(x_{1}, t\right)\right]+\left(\frac{1}{16}\right) \operatorname{Var}\left[\beta\left(x_{1}, t\right)\right]\right.\right. \\
& \left.\left.+\left(\frac{3}{8}\right) \operatorname{Cov}\left[\alpha\left(x_{1}, t\right), \beta\left(x_{1}, t\right)\right]\right]\right\}
\end{aligned}
$$




$$
\begin{aligned}
& +\frac{\partial^{2}}{\partial x_{2}^{2}}\left\{P \left[\left(\frac{1}{16}\right) \operatorname{Var}\left[\alpha\left(x_{2}, t\right)\right]+\left(\frac{9}{16}\right) \operatorname{Var}\left[\beta\left(x_{2}, t\right)\right]\right.\right. \\
& \left.\left.+\left(\frac{3}{8}\right) \operatorname{Cov}\left[\alpha\left(x_{2}, t\right) \beta\left(x_{2}, t\right)\right]\right]\right\} \\
& +\frac{\partial^{2}}{\partial \alpha^{2}}\left\{P \left[\frac { g ^ { 2 } } { 1 6 k ^ { 4 } } ( \frac { 2 } { b } ) ^ { 8 / 3 } \operatorname { V a r } \left[n^{2}\left(x_{1}, t\right)\right.\right.\right. \\
& \left.\left.\left.\left.\cdot\left[\alpha\left(x_{1}, t\right)+\beta\left(x_{1}, t\right)\right]^{2} \cdot\left\{\frac{8 g b}{\left[\alpha\left(x_{1}, t\right)-\beta\left(x_{1}, t\right)\right]^{2}}+1\right\}\right\}^{4 / 3}\right]\right]\right\} \\
& +\frac{\partial^{2}}{\partial \beta^{2}}\left\{P \left[\frac { g ^ { 2 } } { 1 6 k ^ { 4 } } ( \frac { 2 } { b } ) ^ { 8 / 3 } \operatorname { V a r } \left[n^{2}\left(x_{2}, t\right)\right.\right.\right. \\
& \left.\left.\left.\cdot\left[\alpha\left(x_{2}, t\right)+\beta\left(x_{2}, t\right)\right]^{2} \cdot\left\{\frac{8 g b}{\left[\alpha\left(x_{2}, t\right)-\beta\left(x_{2}, t\right)\right]^{2}}+1\right\}^{4 / 3}\right]\right]\right\} .
\end{aligned}
$$

Denoting the advection terms with $F$ and the diffusion terms with $D$, Eq. (25) can be written in a simplified form as follows:

$$
\begin{aligned}
& \frac{\partial P\left(x_{1}, x_{2}, \alpha, \beta ; t\right)}{\partial t}=-\frac{\partial}{\partial x_{1}} F_{1} P-\frac{\partial}{\partial x_{2}} F_{2} P-\frac{\partial}{\partial \alpha} F_{\alpha} P-\frac{\partial}{\partial \beta} F_{\beta} P \\
& +\frac{\partial^{2}}{\partial x_{1}^{2}} D_{1} P+\frac{\partial^{2}}{\partial x_{2}^{2}} D_{2} P+\frac{\partial^{2}}{\partial \alpha^{2}} D_{\alpha} P+\frac{\partial^{2}}{\partial \beta^{2}} D_{\beta} P
\end{aligned}
$$

Equation (26) is the final analytical form of the FPE methodology proposed in this study for the probabilistic solution of the stochastic Saint-Venant equations in one simulation. The advection-diffusion form of Eq. (26) is clear, in which the $F$ terms are the advection coefficients, and the $D$ terms are their corresponding diffusion coefficients. With the mathematical equations for the FPE methodology being derived, the next step is to find a numerical scheme with which Eq. (26) may be computed.

\subsection{Numerical solution for the proposed Fokker-Planck equation methodology}

In order to apply the derived FPE methodology, the FPE represented in Eq. (26) must be solved using an appropriate numerical scheme. In general, finite-difference and finite-element methods, among others, have been widely used to solve FPEs numerically. Many studies have compared several such methods to determine how they perform against each other in solving different FPEs. In their study, Pichler et al. (2011) looked at the central finite-difference method, the alternating directions implicit (ADI) method, as well as finite-element methods. They mentioned that finitedifference methods are computationally more economical than finite-element methods as the number of dimensions increases. Park and Petrosian (1996) performed a comparison between the Chang and Cooper (1970) scheme, the schemes presented in Larsen et al. (1985), and some implicit schemes (including a fully implicit mid-point difference method). They also studied the semi-implicit forms of these schemes (e.g., the Crank-Nicholson scheme). They concluded that, among these schemes, the best finite-difference method for solving their FPEs was the Chang-Cooper scheme, as it was the most robust and most stable over a wide range of parameters among the other methods tested in their study.

In fact, the Chang-Cooper scheme has been cited as one of the most widely known schemes for solving the classical FPE numerically. In their paper, Chang and Cooper (1970) developed a practical numerical differencing scheme for the solution of the one-dimensional classical FPE. This scheme uses a centered difference for the diffusion term, a weighted difference for the advection term, and requires that the quasi-equilibrium solution to the FPE be satisfied exactly at any given time along the mesh nodes. The Chang-Cooper scheme, which has first-order convergence in space and time, is a widely used scheme that ensures the non-negativity of the solution, the conservation of the probability mass (in the absence of any external sources or sinks), and the exact representation of the analytical solution upon equilibration. As a result, the Chang-Cooper scheme is highly accurate with a relatively small number of required mesh nodes. While Chang and Cooper (1970) developed and applied that scheme to a one-dimensional FPE, Kim et al. (2005a) generalized and applied the Chang-Cooper scheme to a two-dimensional FPE. In a similar manner, for the case of this study, an attempt is made to generalize the Chang-Cooper scheme to the four-dimensional FPE shown in Eq. (26).

First, Eq. (26) is rewritten as follows:

$$
\begin{aligned}
& \frac{\partial P\left(x_{1}, x_{2}, \alpha, \beta ; t\right)}{\partial t}=-\frac{\partial}{\partial x_{1}}\left[F_{1} P-D_{1} \frac{\partial}{\partial x_{1}} P\right] \\
& -\frac{\partial}{\partial x_{2}}\left[F_{2} P-D_{2} \frac{\partial}{\partial x_{2}} P\right]-\frac{\partial}{\partial \alpha}\left[F_{\alpha} P-D_{\alpha} \frac{\partial}{\partial \alpha} P\right] \\
& -\frac{\partial}{\partial \beta}\left[F_{\beta} P-D_{\beta} \frac{\partial}{\partial \beta} P\right] \\
& =-\frac{\partial}{\partial x_{1}} J_{1}-\frac{\partial}{\partial x_{2}} J_{2}-\frac{\partial}{\partial \alpha} J_{\alpha}-\frac{\partial}{\partial \beta} J_{\beta} .
\end{aligned}
$$

Equation (27) is in the form of the continuity equation, in which the $J$ parameters may be interpreted as the probability flux or probability current, whereas $P$ (i.e., the probability density function) is considered as the state variable. This equation can then be discretized in the following implicit manner:

$$
\begin{aligned}
\frac{P_{i, j, k, l}^{n+1}-P_{i, j, k, l}^{n}}{\Delta t} & =-\frac{J_{1 ; i+\frac{1}{2}, j, k, l}^{n+1}-J_{1 ; i-\frac{1}{2}, j, k, l}^{n+1}}{\Delta x_{1}} \\
& -\frac{J_{2 ; i, j+\frac{1}{2}, k, l}^{n+1}-J_{2 ; i, j-\frac{1}{2}, k, l}^{n+1}}{\Delta x_{2}} \\
& -\frac{J_{\alpha ; i, j, k+\frac{1}{2}, l}^{n+1}-J_{\alpha ; i, j, k-\frac{1}{2}, l}^{n+1}}{\Delta \alpha} \\
& -\frac{J_{\beta ; i, j, k, l+\frac{1}{2}}^{n+1}-J_{\beta ; i, j, k, l-\frac{1}{2}}^{n+1}}{\Delta \beta}
\end{aligned}
$$


where $i: 0,1,2, \ldots, N_{I}$ denotes the domain of $x_{1}$ in the direction of the $C_{1}$ curve; $j: 0,1,2, \ldots, N_{J}$ denotes the domain of $x_{2}$ in the direction of the $C_{2}$ curve; $k: 0,1,2, \ldots, N_{K}$ denotes the domain of $\alpha ; l: 0,1,2, \ldots, N_{L}$ denotes the domain of $\beta$; and $n: 0,1,2, \ldots$ denotes the domain of time $t$. Following the Chang-Cooper scheme, the expression for computing variables between two nodes (e.g., at $i+1 / 2$ ) is defined by the following expression, which is an analogue for the other dimensions:

$$
P_{i+\frac{1}{2}, j, k, l}^{n+1}=\left(1-\lambda_{1 ; i}^{n+1}\right) P_{i+1, j, k, l}^{n+1}+\lambda_{1 ; i}^{n+1} P_{i, j, k, l}^{n+1},
$$

where $\lambda$ is a weighting factor. In the one-dimensional case, Chang and Cooper (1970) developed the expression of the weighting factor in a manner that would ensure the nonnegativity of the PDF solution and that would give proper equilibration. In a similar manner, the same steps are followed for this study in order to derive the expression for $\lambda$. This expression, corresponding to Eq. (29), is shown below and is an analogue for the other dimensions:

$$
\begin{aligned}
& \lambda_{1 ; i}^{n+1}= \\
& \frac{D_{1 ; i+\frac{1}{2}, j, k, l}^{n}-\left(D_{1 ; i+\frac{1}{2}, j, k, l}^{n}-\Delta x_{1} F_{1 ; i+\frac{1}{2}, j, k, l}^{n}\right) \exp \left[\Delta x_{1} F_{\left.\frac{1 ; i+\frac{1}{2}, j, k, l}{D_{1 ; i+\frac{1}{2}, j, k, l}^{n}}\right]}\right.}{\Delta x_{1} F_{1 ; i+\frac{1}{2}, j, k, l}^{n}\left\{\exp \left[\Delta x_{1} F_{1 ; i \frac{1}{2}, j, k, l}^{n} D_{1 ; i+\frac{1}{2}, j, k, l}^{n}\right]-1\right\}}
\end{aligned}
$$

Moreover, following the Chang-Cooper scheme, the expression for the $J$ parameter in the $x_{1}$ direction may be derived to be represented as follows:

$$
\begin{aligned}
& J_{1 ; i+\frac{1}{2}, j, k, l}^{n+1}=\left[F_{1 ; i+\frac{1}{2}, j, k, l}^{n}\left(1-\lambda_{1 ; i}^{n+1}\right)-\frac{D_{1 ; i+\frac{1}{2}, j, k, l}^{n}}{\Delta x_{1}}\right] P_{i+1, j, k, l}^{n+1} \\
& +\left[F_{1 ; i+\frac{1}{2}, j, k, l}^{n} \lambda_{1 ; i}^{n+1}+\frac{D_{1 ; i+\frac{1}{2}, j, k, l}^{n}}{\Delta x_{1}}\right] P_{i, j, k, l}^{n+1} .
\end{aligned}
$$

Equation (31), and its analogous equations for the other dimensions, can then be substituted into the discretized FPE, Eq. (28), in order to provide the implicit finite-difference form of the FPE methodology, shown in Eq. (32) below, which can be numerically solved. Note that in the expression of Eq. (32), each subscript $(i, j, k, l)$ that is not followed by a $+1 / 2$ or $-1 / 2$ is dropped from the expressions of $F$ and $D$ for readability purposes; e.g., $F_{2 ; i, j+\frac{1}{2}, k, l}^{n}$ is simplified and written as $F_{2 ; j+\frac{1}{2}}^{n}$

$$
\begin{aligned}
& P_{i, j, k, l}^{n}=\left\{\begin{array}{l}
1+\frac{\Delta t}{\Delta x_{1}} \lambda_{1 ; i}^{n+1} F_{1 ; i+\frac{1}{2}}^{n}+\frac{\Delta t}{\left(\Delta x_{1}\right)^{2}} D_{1 ; i+\frac{1}{2}}^{n} \\
-\frac{\Delta t}{\Delta x_{1}}\left(1-\lambda_{1 ; i-1}^{n+1}\right) F_{1 ; i-\frac{1}{2}}^{n}+\frac{\Delta t}{\left(\Delta x_{1}\right)^{2}} D_{1 ; i-\frac{1}{2}}^{n} \\
+\frac{\Delta t}{\Delta x_{2}} \lambda_{2 ; j}^{n+1} F_{2 ; j+\frac{1}{2}}^{n}+\frac{\Delta t}{\left(\Delta x_{2}\right)^{2}} D_{2 ; j+\frac{1}{2}}^{n} \\
-\frac{\Delta t}{\Delta x_{2}}\left(1-\lambda_{2 ; j-1}^{n+1}\right) F_{2 ; j-\frac{1}{2}}^{n}+\frac{\Delta t}{\left(\Delta x_{2}\right)^{2}} D_{2 ; j-\frac{1}{2}}^{n} \\
+\frac{\Delta t}{\Delta \alpha} \lambda_{\alpha ; k}^{n+1} F_{\alpha ; k+\frac{1}{2}}^{n}+\frac{\Delta t}{(\Delta \alpha)^{2}} D_{\alpha ; k+\frac{1}{2}}^{n} \\
-\frac{\Delta t}{\Delta \alpha}\left(1-\lambda_{\alpha ; k-1}^{n+1}\right) F_{\alpha ; k-\frac{1}{2}}^{n}+\frac{\Delta t}{(\Delta \alpha)^{2}} D_{\alpha ; k-\frac{1}{2}}^{n} \\
+\frac{\Delta t}{\Delta \beta} \lambda_{\beta ; l}^{n+1} F_{\beta ; l+\frac{1}{2}}^{n}+\frac{\Delta t}{(\Delta \beta)^{2}} D_{\beta ; l+\frac{1}{2}}^{n} \\
-\frac{\Delta t}{\Delta \beta}\left(1-\lambda_{\beta ; l-1}^{n+1}\right) F_{\beta ; l-\frac{1}{2}}^{n}+\frac{\Delta t}{(\Delta \beta)^{2}} D_{\beta ; l-\frac{1}{2}}^{n}
\end{array}\right\} P_{i, j, k, l}^{n+1} \\
& +\left[\frac{\Delta t}{\Delta x_{1}}\left(1-\lambda_{1 ; i}^{n+1}\right) F_{1 ; i+\frac{1}{2}}^{n}-\frac{\Delta t}{\left(\Delta x_{1}\right)^{2}} D_{1 ; i+\frac{1}{2}}^{n}\right] P_{i+1, j, k, l}^{n+1} \\
& +\left[\frac{\Delta t}{\Delta x_{2}}\left(1-\lambda_{2 ; j}^{n+1}\right) F_{2 ; j+\frac{1}{2}}^{n}-\frac{\Delta t}{\left(\Delta x_{2}\right)^{2}} D_{2 ; j+\frac{1}{2}}^{n}\right] P_{i, j+1, k, l}^{n+1} \\
& +\left[\frac{\Delta t}{\Delta \alpha}\left(1-\lambda_{\alpha ; k}^{n+1}\right) F_{\alpha ; k+\frac{1}{2}}^{n}-\frac{\Delta t}{(\Delta \alpha)^{2}} D_{\alpha ; k+\frac{1}{2}}^{n}\right] P_{i, j, k+1, l}^{n+1} \\
& +\left[\frac{\Delta t}{\Delta \beta}\left(1-\lambda_{\beta ; l}^{n+1}\right) F_{\beta ; l+\frac{1}{2}}^{n}-\frac{\Delta t}{(\Delta \beta)^{2}} D_{\beta ; l+\frac{1}{2}}^{n}\right] P_{i, j, k, l+1}^{n+1} \\
& +\left[-\frac{\Delta t}{\Delta x_{1}} \lambda_{1 ; i-1}^{n+1} F_{1 ; i-\frac{1}{2}}^{n}-\frac{\Delta t}{\left(\Delta x_{1}\right)^{2}} D_{1 ; i-\frac{1}{2}}^{n}\right] P_{i-1, j, k, l}^{n+1} \\
& +\left[-\frac{\Delta t}{\Delta x_{2}} \lambda_{2 ; j-1}^{n+1} F_{2 ; j-\frac{1}{2}}^{n}-\frac{\Delta t}{\left(\Delta x_{2}\right)^{2}} D_{2 ; j-\frac{1}{2}}^{n}\right] P_{i, j-1, k, l}^{n+1} \\
& +\left[-\frac{\Delta t}{\Delta \alpha} \lambda_{\alpha ; k-1}^{n+1} F_{\alpha ; k-\frac{1}{2}}^{n}-\frac{\Delta t}{(\Delta \alpha)^{2}} D_{\alpha ; k-\frac{1}{2}}^{n}\right] P_{i, j, k-1, l}^{n+1} \\
& +\left[-\frac{\Delta t}{\Delta \beta} \lambda_{\beta ; l-1}^{n+1} F_{\beta ; l-\frac{1}{2}}^{n}-\frac{\Delta t}{(\Delta \beta)^{2}} D_{\beta ; l-\frac{1}{2}}^{n}\right] P_{i, j, k, l-1}^{n+1}
\end{aligned}
$$

Note that the derived FPE discretized in Eq. (32) was originally described and represented using the characteristic method. As a result, the computed values of the state variables at a new time step would be solved at the intersection of the characteristic curves $C_{1}$ and $C_{2}$ (see Eqs. 3 and 5). In a similar manner, the values of $P$ to be solved for in Eq. (32) should be those corresponding to the positions of intersection between $C_{1}$ and $C_{2}$, i.e., when $x_{1}=x_{2}$. Hence, additional simplifications can be applied to Eq. (32), including $x_{1}=x_{2}=x$ and $\Delta x_{1}=\Delta x_{2}=\Delta x$. As such, since the variables $x_{1}$ and $x_{2}$ are now represented by one variable $x$, which is the intersection position, their corresponding $i$ and $j$ subscript representations can be merged into a single representation, $h$, thus reducing the equation from four to three dimensions $(x, \alpha, \beta)$. Therefore, the PDF $P_{i, j, k, l}$ can now be represented as $P_{h, k, l}$, where $h$ represents the domain of the intersection position $x$. However, note that this does not affect the computations of the $F, D$, and $\lambda$ parameters for $x_{1}$ and $x_{2}$ since each one has its own different expression for its calculation. With the above changes, Eq. (32) can finally be rewritten as shown in Eq. (33). 


$$
\begin{aligned}
& P_{h, k, l}^{n}=\left\{\begin{array}{l}
1+\frac{\Delta t}{\Delta x} \lambda_{1 ; h}^{n+1} F_{1 ; h+\frac{1}{2}}^{n}+\frac{\Delta t}{(\Delta x)^{2}} D_{1 ; h+\frac{1}{2}}^{n} \\
-\frac{\Delta t}{\Delta x}\left(1-\lambda_{1 ; h-1}^{n+1}\right) F_{1 ; h-\frac{1}{2}}^{n}+\frac{\Delta t}{(\Delta x)^{2}} D_{1 ; h-\frac{1}{2}}^{n} \\
+\frac{\Delta t}{\Delta x} \lambda_{2 ; h}^{n+1} F_{2 ; h+\frac{1}{2}}^{n}+\frac{\Delta t}{(\Delta x)^{2}} D_{2 ; h+\frac{1}{2}}^{n} \\
-\frac{\Delta t}{\Delta x}\left(1-\lambda_{2 ; h-1}^{n+1}\right) F_{2 ; h-\frac{1}{2}}^{n}+\frac{\Delta t}{(\Delta x)^{2}} D_{2 ; h-\frac{1}{2}}^{n} \\
+\frac{\Delta t}{\Delta \alpha} \lambda_{\alpha ; k}^{n+1} F_{\alpha ; k+\frac{1}{2}}^{n}+\frac{\Delta t}{(\Delta \alpha)^{2}} D_{\alpha ; k+\frac{1}{2}}^{n} \\
-\frac{\Delta t}{\Delta \alpha}\left(1-\lambda_{\alpha ; k-1}^{n+1}\right) F_{\alpha ; k-\frac{1}{2}}^{n}+\frac{\Delta t}{(\Delta \alpha)^{2}} D_{\alpha ; k-\frac{1}{2}}^{n} \\
+\frac{\Delta t}{\Delta \beta} \lambda_{\beta ; l}^{n+1} F_{\beta ; l+\frac{1}{2}}^{n}+\frac{\Delta t}{(\Delta \beta)^{2}} D_{\beta ; l+\frac{1}{2}}^{n} \\
-\frac{\Delta t}{\Delta \beta}\left(1-\lambda_{\beta ; l-1}^{n+1}\right) F_{\beta ; l-\frac{1}{2}}^{n}+\frac{\Delta t}{(\Delta \beta)^{2}} D_{\beta ; l-\frac{1}{2}}^{n}
\end{array}\right\} P_{h, k, l}^{n+1} \\
& +\left\{\begin{array}{c}
\frac{\Delta t}{\Delta x}\left(1-\lambda_{1 ; h}^{n+1}\right) F_{1 ; h+\frac{1}{2}}^{n}-\frac{\Delta t}{(\Delta x)^{2}} D_{1 ; h+\frac{1}{2}}^{n} \\
+\frac{\Delta t}{\Delta x}\left(1-\lambda_{2 ; h}^{n+1}\right) F_{2 ; h+\frac{1}{2}}^{n}-\frac{\Delta t}{(\Delta x)^{2}} D_{2 ; h+\frac{1}{2}}^{n}
\end{array}\right\} P_{h+1, k, l}^{n+1} \\
& +\left[\frac{\Delta t}{\Delta \alpha}\left(1-\lambda_{\alpha ; k}^{n+1}\right) F_{\alpha ; k+\frac{1}{2}}^{n}-\frac{\Delta t}{(\Delta \alpha)^{2}} D_{\alpha ; k+\frac{1}{2}}^{n}\right] P_{h, k+1, l}^{n+1} \\
& +\left[\frac{\Delta t}{\Delta \beta}\left(1-\lambda_{\beta ; l}^{n+1}\right) F_{\beta ; l+\frac{1}{2}}^{n}-\frac{\Delta t}{(\Delta \beta)^{2}} D_{\beta ; l+\frac{1}{2}}^{n}\right] P_{h, k, l+1}^{n+1} \\
& +\left\{\begin{array}{l}
-\frac{\Delta t}{\Delta x} \lambda_{1 ; h-1}^{n+1} F_{1 ; h-\frac{1}{2}}^{n}-\frac{\Delta t}{(\Delta x)^{2}} D_{1 ; h-\frac{1}{2}}^{n} \\
-\frac{\Delta t}{\Delta x} \lambda_{2 ; h-1}^{n+1} F_{2 ; h-\frac{1}{2}}^{n}-\frac{\Delta t}{(\Delta x)^{2}} D_{2 ; h-\frac{1}{2}}^{n}
\end{array}\right\} P_{h-1, k, l}^{n+1} \\
& +\left[-\frac{\Delta t}{\Delta \alpha} \lambda_{\alpha ; k-1}^{n+1} F_{\alpha ; k-\frac{1}{2}}^{n}-\frac{\Delta t}{(\Delta \alpha)^{2}} D_{\alpha ; k-\frac{1}{2}}^{n}\right] P_{h, k-1, l}^{n+1} \\
& +\left[-\frac{\Delta t}{\Delta \beta} \lambda_{\beta ; l-1}^{n+1} F_{\beta ; l-\frac{1}{2}}^{n}-\frac{\Delta t}{(\Delta \beta)^{2}} D_{\beta ; l-\frac{1}{2}}^{n}\right] P_{h, k, l-1}^{n+1}
\end{aligned}
$$

Equation (33) is the discretized version of the FPE that represents the proposed methodology of this study. A comparison of Eqs. (25) and (26) provides the expressions for the $F$ and $D$ parameters, while Eq. (30) and its analogues for the other dimensions provide the expressions for the $\lambda$ parameters, all of which would then complete the solution of Eq. (33). This equation has to be solved implicitly in order to compute the ensemble behavior and variability of a hydrologic system defined by the stochastic Saint-Venant equations, and it does that by solving for the joint PDF of the state variables within the $x-\alpha-\beta$ domain. Equation (33) provides an effective approach to solve for the ensemble behavior and variability of the stochastic unsteady open-channel flow in a rectangular, prismatic channel under uncertain roughness coefficient, by running only one simulation. This proposed FPE methodology can also be expanded to problems with uncertainties in other channel or flow parameters. The performance of the proposed FPE methodology is evaluated in a companion paper by Dib and Kavvas (2018) which compares its results to those obtained by the MC approach.

\section{Summary and conclusions}

This study proposed a new methodology to model the expected behavior and variability of a system described by the stochastic open-channel flow equations. The governing equations that were used to represent the flood routing problem in this study are the continuity and momentum equations, otherwise known as the Saint-Venant equations. Many uncertainties can add to the complexity of solving the Saint-Venant equations in engineering routing problems. These uncertainties may include uncertainties in the channel's physical and geometric properties, as well as uncertainties in the lateral inflows and upstream boundary conditions, all of which render the Saint-Venant equations stochastic. As such, the dependent variables that will be solved for by these equations will also become stochastic, thus requiring that their statistical properties be solved for at specific time-space locations. Therefore, with uncertain parameters, the Saint-Venant equations have to be solved within a stochastic framework in order to quantify the ensemble behavior and variability of the system being considered. While the Mote Carlo method is a viable approach for the solution of such a stochastic unsteady open-channel flow problem, its computational expense and its large number of simulations act to its disadvantage. Hence, a new methodology was proposed in this study by which the statistical properties of the dependent variables of the considered hydrologic problem may be obtained in only one single simulation.

The proposed FPE methodology derived in this study involved upscaling the governing stochastic differential equations by developing their corresponding LagrangianEulerian Fokker-Planck Equation (LEFPE), thus transforming the original stochastic equations into the framework of a deterministic differential equation. The deterministic LEFPE that describes the time-space evolution of the probability density function of the unsteady open-channel flow state variables, was developed following the method in Kavvas (2003) after the governing Saint Venant equations were transformed into their characteristic form by using the method of characteristics. Through simplifications, this LEFPE was reduced to a classical FPE that could be solved deterministically for the evolution of the probability density of the state variables of the system. The obtained linear FPE was, then, discretized in an implicit manner following Chang and Cooper (1970). This provided the equations that may be solved numerically, through only one simulation, in order to determine the ensemble behavior and variability of a system described by the stochastic open-channel flow equations. The application and validation of this methodology, applied to an open-channel flow problem with an uncertain roughness coefficient, is provided in a companion paper by Dib and Kavvas (2018), in which the statistical results of the proposed FPE methodology are compared against the results obtained by the MC approach. 
The open-channel flow problem considered in this study was for a rectangular, prismatic channel under an uncertain roughness coefficient. However, the proposed methodology can be expanded to problems which assume uncertainties that arise from other flow or channel parameters. For instance, when parameters such as the channel bed slope or the channel width are assumed to be uncertain, their corresponding representations in the equations will simply have to be included in the expectation and variance expressions of the advection and diffusion coefficients for the $\alpha$ and $\beta$ directions. When lateral inflows/outflows exist and are uncertain, additional terms corresponding to the lateral inflows/outflows will have to be added to the Saint-Venant equations, and will subsequently appear in the derived FPE. These additional terms will also have to be included inside the expectation and variance expressions. Hence, future research could entail investigating the uncertainties due to the channel slope, channel cross section, lateral inflows, and initial and boundary conditions. Moreover, any number of such uncertainties may be incorporated into this methodology at the same time by simultaneously applying their corresponding changes to the necessary equations. Therefore, applying the proposed FPE methodology to systems which include more than one source of uncertainty could be a further extension of the methodology in its attempt to effectively describe such highly nonlinear and stochastic systems.

Data availability. This study involved the theoretical development and derivation of a new proposed methodology for the stochastic solution of unsteady open-channel flow. No data were used in the derivation process.

Competing interests. The authors declare that they have no conflict of interest.

Edited by: Sabine Attinger

Reviewed by: two anonymous referees

\section{References}

Abbott, M. B.: An introduction to the method of characteristics, American Elsevier, New York, 243 pp., 1966.

Abbott, M. B. and Ionescu, F.: On the numerical computation of nearly horizontal flows, J. Hydraul. Res., 5, 97-117, https://doi.org/10.1080/00221686709500195, 1967.

Amein, M.: Streamflow routing on computer by characteristics, Water Resour. Res., 2, 123-130, https://doi.org/10.1029/WR002i001p00123, 1966.

Barré de Saint-Venant, A.-J.-C.: Théorie du mouvement non permanent des eaux, avec application aux crues des rivières et à l'introduction des marées dans leur lit, C. R. Acad. Sci., 73, 147154,1871

Beam, R. M. and Warming, R. F.: Implicit finite-difference algorithm for hyperbolic systems in conservation-law form,
J. Comput. Phys., 22, 87-110, https://doi.org/10.1016/00219991(76)90110-8, 1976.

Bellin, A., Salandin, P., and Rinaldo, A.: Simulation of dispersion in heterogeneous porous formations - statistics, 1st-order theories, convergence of computations, Water Resour. Res., 28, 22112227, https://doi.org/10.1029/92wr00578, 1992.

Cayar, M. and Kavvas, M. L.: Symmetry in nonlinear hydrologic dynamics under uncertainty: ensemble modeling of $2 \mathrm{~d}$ Boussinesq equation for unsteady flow in heterogeneous aquifers, J. Hydrol. Eng., 14, 1173-1184, https://doi.org/10.1061/(Asce)He.1943-5584.0000112, 2009a.

Cayar, M. and Kavvas, M. L.: Ensemble average and ensemble variance behavior of unsteady, one-dimensional groundwater flow in unconfined, heterogeneous aquifers: an exact second-order model, Stoch. Env. Res. Risk. A., 23, 947-956, https://doi.org/10.1007/s00477-008-0263-1, 2009b.

Chang, J. S. and Cooper, G.: A practical difference scheme for Fokker-Planck equations, J. Comput. Phys., 6, 1-16, https://doi.org/10.1016/0021-9991(70)90001-X, 1970.

Chanson, H.: The hydraulics of open channel flow: an introduction, in: 2nd Edn., Butterworth-Heinemann, Oxford, 585 pp., 2004.

Chaudhry, M. H.: Open-channel flow, in: 2nd Edn., Springer US, New York, 523 pp., 2008.

Chow, V. T.: Open-channel hydraulics, McGraw-Hill civil engineering series, McGraw-Hill, New York, 680 pp., 1959.

Cooley, R. L. and Moin, S. A.: Finite-element solution of SaintVenant equations, J. Hydrol. Eng. Div.-Asce, 102, 759-775, 1976.

Dettinger, M. D. and Wilson, J. L.: 1st-order analysis of uncertainty in numerical-models of groundwater-flow .1. mathematical development, Water Resour. Res., 17, 149-161, https://doi.org/10.1029/WR017i001p00149, 1981.

Dib, A. and Kavvas, M. L.: Ensemble modeling of stochastic unsteady open-channel flow in terms of its time-space evolutionary probability distribution - Part 2: numerical application, Hydrol. Earth Syst. Sci., 22, 2007-2021, https://doi.org/10.5194/hess-222007-2018, 2018.

Dogrul, E. C., Kavvas, M. L., and Chen, Z. Q.: Prediction of subsurface stormflow in heterogeneous sloping aquifers, J. Hydrol. Eng., 3, 258-267, https://doi.org/10.1061/(Asce)10840699(1998)3:4(258), 1998.

Duffy, C. J.: A two-state integral-balance model for soil moisture and groundwater dynamics in complex terrain, Water Resour Res., 32, 2421-2434, https://doi.org/10.1029/96wr01049, 1996.

Duffy, C. J. and Cusumano, J.: A low-dimensional model for concentration-discharge dynamics in groundwater stream systems, Water Resour. Res., 34, 2235-2247, https://doi.org/10.1029/98wr01705, 1998.

Ercan, A. and Kavvas, M. L.: Ensemble modeling of hydrologic and hydraulic processes at one shot: application to kinematic openchannel flow under uncertain channel properties by the stochastic method of characteristics, J. Hydrol. Eng., 17, 168-181, https://doi.org/10.1061/(Asce)He.1943-5584.0000425, 2012a.

Ercan, A. and Kavvas, M. L.: Ensemble modeling of hydrologic and hydraulic processes at one shot: application to kinematic open-channel flow under uncertain channel properties and uncertain lateral flow conditions by the stochastic method of characteristics, J. Hydrol. Eng., 17, 414-423, https://doi.org/10.1061/(Asce)He.1943-5584.0000434, 2012b. 
Fennema, R. J. and Chaudhry, M. H.: Explicit numerical schemes for unsteady free-surface flows with shocks, Water Resour. Res., 22, 1923-1930, https://doi.org/10.1029/WR022i013p01923, 1986.

Fread, D. L.: Technique for implicit dynamic routing in rivers with tributaries, Water Resour. Res., 9, 918-926, https://doi.org/10.1029/WR009i004p00918, 1973.

Freeze, R. A.: Stochastic-conceptual analysis of onedimensional groundwater flow in nonuniform homogeneous media, Water Resour. Res., 11, 725-741, https://doi.org/10.1029/WR011i005p00725, 1975.

Garcia, R. and Kahawita, R. A.: Numerical-solution of the St-Venant equations with the Maccormack finitedifference scheme, Int. J. Numer. Meth. Fluids, 6, 259-274, https://doi.org/10.1002/fld.1650060502, 1986.

Gates, T. K. and AlZahrani, M. A.: Spatiotemporal stochastic open-channel flow. 1. model and its parameter data, J. Hydraul. Eng., 122, 641-651, https://doi.org/10.1061/(Asce)07339429(1996)122:11(641), 1996a.

Gates, T. K. and AlZahrani, M. A.: Spatiotemporal stochastic open-channel flow. 2. simulation experiments, J. Hydraul. Eng., 122, 652-661, https://doi.org/10.1061/(Asce)07339429(1996)122:11(652), 1996b.

Gelhar, L. W. and Axness, C. L.: 3-dimensional stochastic-analysis of macrodispersion in aquifers, Water Resour. Res., 19, 161-180, https://doi.org/10.1029/WR019i001p00161, 1983.

Hicks, F. E. and Steffler, P. M.: Comparison of finite-element methods for the St Venant equations, Int. J. Numer. Meth. Fluids, 20, 99-113, https://doi.org/10.1002/fld.1650200202, 1995.

Hoffman, J. D.: Numerical methods for engineers and scientists, in: 2nd Edn., Marcel Dekker, New York, 823 pp., 2001.

Horne, F. E. and Kavvas, M. L.: Physics of the spatially averaged snowmelt process, J. Hydrol., 191, 179-207, https://doi.org/10.1016/S0022-1694(96)03063-6, 1997.

Kapoor, V. and Gelhar, L. W.: Transport in 3-dimensionally heterogeneous aquifers. 1. dynamics of concentration fluctuations, Water Resour. Res., 30, 1775-1788, https://doi.org/10.1029/94wr00076, 1994.

Kavvas, M. L.: Nonlinear hydrologic processes: conservation equations for determining their means and probability distributions, J. Hydrol. Eng., 8, 44-53, https://doi.org/10.1061/(Asce)10840699(2003)8:2(44), 2003.

Kavvas, M. L. and Karakas, A.: On the stochastic theory of solute transport by unsteady and steady groundwater flow in heterogeneous aquifers, J. Hydrol., 179, 321-351, 1996.

Kavvas, M. L. and Wu, J.: Conservation equations for solute transport by unsteady and steady flows in heterogeneous aquifers: the cumulant expansion/Lie operator methodology, in: Stochastic Methods in Subsurface Contaminant Hydrology, edited by: Govindaraju, R. S., ASCE Press, New York, 281-306, 2002.

Kim, S., Kavvas, M. L., and Chen, Z. Q.: Root-water uptake model at heterogeneous soil fields, J. Hydrol. Eng., 10, 160 167, https://doi.org/10.1061/(Asce)1084-0699(2005)10:2(160), 2005a.

Kim, S., Kavvas, M. L., and Yoon, J.: Upscaling of vertical unsaturated flow model under infiltration condition, J. Hydrol. Eng., 10, 151-159, https://doi.org/10.1061/(Asce)10840699(2005)10:2(151), 2005b.
Kitanidis, P. K.: Prediction by the method of moments of transport in a heterogeneous formation, J. Hydrol., 102, 453-473, https://doi.org/10.1016/0022-1694(88)90111-4, 1988.

Lai, C.: Comprehensive method of characteristics models for flow simulation, J. Hydraul. Eng., 114, 1074-1097, 1988.

Larsen, E. W., Levermore, C. D., Pomraning, G. C., and Sanderson, J. G.: Discretization Methods for One-Dimensional Fokker-Planck Operators, J. Comput. Phys., 61, 359-390, https://doi.org/10.1016/0021-9991(85)90070-1, 1985.

Liang, L. and Kavvas, M. L.: Modeling of solute transport and macrodispersion by unsteady stream flow under uncertain conditions, J. Hydrol. Eng., 13, 510-520, https://doi.org/10.1061/(Asce)1084-0699(2008)13:6(510), 2008.

Litrico, X. and Fromion, V.: Modeling and control of hydrosystems, Springer, Dordrecht, New York, 409 pp., 2009.

Mantoglou, A. and Gelhar, L. W.: Capillary tension head variance, mean soil-moisture content, and effective specific soil-moisture capacity of transient unsaturated flow in stratified soils, Water Resour. Res., 23, 47-56, https://doi.org/10.1029/WR023i001p00047, 1987.

Ohara, N., Kavvas, M. L., and Chen, Z. Q.: Stochastic upscaling for snow accumulation and melt processes with PDF approach, J. Hydrol. Eng., 13, 1103-1118, https://doi.org/10.1061/(Asce)1084-0699(2008)13:12(1103), 2008.

Park, B. T. and Petrosian, V.: Fokker-Planck equations of stochastic acceleration: A study of numerical methods, Astrophys. J. Suppl. S., 103, 255-267, https://doi.org/10.1086/192278, 1996.

Pichler, L., Masud, A., and Bergman, L. A.: Numerical Solution of the Fokker-Planck Equation by Finite Difference and Finite Element Methods - A Comparative Study, in: Computational Methods in Stochastic Dynamics, edited by: Papadrakakis, M., Stefanou, G., and Papdopoulos, V., Springer, Dordrecht, 2011.

Puente, C. E.: A new approach to hydrologic modeling: derived distributions revisited, J. Hydrol., 187, 65-80, https://doi.org/10.1016/S0022-1694(97)87978-4, 1996.

Rubin, Y. and Dagan, G.: Stochastic-analysis of boundaries effects on head spatial variability in heterogeneous aquifers. 2. impervious boundary, Water Resour. Res., 25, 707-712, https://doi.org/10.1029/WR025i004p00707, 1989.

Scharffenberg, W. A. and Kavvas, M. L.: Uncertainty in flood wave routing in a lateral-inflow-dominated stream, J. Hydrol. Eng., 16, 165-175, https://doi.org/10.1061/(Asce)He.19435584.0000298, 2011.

Serrano, S. E.: Analytical solutions of the nonlinear groundwaterflow equation in unconfined aquifers and the effect of heterogeneity, Water Resour. Res., 31, 2733-2742, https://doi.org/10.1029/95wr02038, 1995.

Smith, L. and Freeze, R. A.: Stochastic-analysis of steadystate groundwater-flow in a bounded domain. 2. twodimensional simulation, Water Resour. Res., 15, 1543-1559, https://doi.org/10.1029/WR015i006p01543, 1979.

Sturm, T. W.: Open channel hydraulics, McGraw-Hill series in water resources and environmental engineering, McGraw-Hill, Boston, 493 pp., 2001.

Szymkiewicz, R.: Finite-element method for the solution of the Saint Venant equations in an open channel network, J. Hydrol., 122, 275-287, https://doi.org/10.1016/0022-1694(91)90182-H, 1991. 
Szymkiewicz, R.: Method to solve 1d unsteady transport and flow equations, J. Hydraul. Eng., 121, 396-403, https://doi.org/10.1061/(Asce)0733-9429(1995)121:5(396), 1995.

Szymkiewicz, R.: Numerical modeling in open channel hydraulics, in: Water science and technology library, 83, Springer, Dordrecht, the Netherlands, 419 pp., 2010.

Tannehill, J. C., Anderson, D. A., and Pletcher, R. H.: Computational fluid mechanics and heat transfer, in: 2nd Edn., Series in computational and physical processes in mechanics and thermal sciences, Taylor \& Francis, Washington, D.C., 792 pp., 1997.

Tayfur, G. and Kavvas, M. L.: Spatially averaged conservation equations for interacting rill-interrill area overland flows, J. Hydraul. Eng., 120, 1426-1448, https://doi.org/10.1061/(Asce)0733-9429(1994)120:12(1426), 1994.

Van Kampen, N. G.: A cumulant expansion for stochastic linear differential equations. II, Physica, 74, 239-247, https://doi.org/10.1016/0031-8914(74)90122-0, 1974.

Van Kampen, N. G.: Stochastic differential equations, Phys. Rep., 24, 171-228, https://doi.org/10.1016/0370-1573(76)90029-6, 1976.
Venutelli, M.: Stability and accuracy of weighted four-point implicit finite difference schemes for open channel flow, J. Hydraul. Eng., 128, 281-288, https://doi.org/10.1061/(Asce)07339429(2002)128:3(281), 2002.

Viessman, W., Knapp, J. W., Lewis, G. L., and Harbaugh, T. E.: Introduction to hydrology, in: 2nd Edn., Series in civil engineering, IEP-Dun-Donnelley, Harper \& Row, New York, 704 pp., 1977.

Wood, B. D. and Kavvas, M. L.: Ensemble-averaged equations for reactive transport in porous media under unsteady flow conditions, Water Resour. Res., 35, 2053-2068, https://doi.org/10.1029/1999wr900113, 1999a.

Wood, B. D. and Kavvas, M. L.: Stochastic solute transport under unsteady flow conditions: Comparison of theory, Monte Carlo simulations, and field data, Water Resour. Res., 35, 2069-2084, https://doi.org/10.1029/1999wr900112, 1999b.

Woolhiser, D. A. and Liggett, J. A.: Unsteady 1-dimensional flow over a plane - rising hydrograph, Water Resour. Res., 3, 753771, https://doi.org/10.1029/WR003i003p00753, 1967. 